\title{
A Novel Bio-inspired Tactile Tumour Detection Concept for Capsule Endoscopy
}

\author{
B Winstone ${ }^{1}$, C Melhuish ${ }^{1}$, S Dogramadzi ${ }^{1}$, T Pipe ${ }^{1}$ M Callaway $^{2}$ \\ ${ }^{1}$ Bristol Robotics Laboratory $\quad{ }^{2}$ Department of Radiology \\ University of the West of England Bristol Royal Infirmary \\ Benjamin.Winstone@brl.ac.uk Mark.Callaway@UHBristol.nhs.uk
}

\section{Introduction}

Examination of the gastrointestinal(GI) tract has traditionally been performed using endoscopy tools that allow a surgeon to see the inside of the lining of the digestive tract. Endoscopes are rigid or flexible tubes that use fibre-optics or cameras to visualise tissues in natural orifices. This can be an uncomfortable and very invasive procedure for the patient.

Modern advances in optical diagnostics have developed wireless capsule endoscopy(WCE) such as the PillCam $\cap$ from Given Imaging, which is a passive imaging system in the form of a large pill swallowed by the patient. WCE is used to inspect the GI tract by use of an internal imaging camera. A capsule endoscopy allows a more comfortable examination for the patient that can reach further in to the GI tract than traditional endoscopy, however with up to 8 fps only and direction being determined by the peristaltic motion of the gut observation of tumours is not guaranteed.

Here we propose using the TACTIP, a tactile sensor to be placed on the outer surface of a WCE so that it can trace along the wall of the intestines during travel through the GI tract. Using established signal processing algorithms the TACTIP can identify raised bumps which deform the surface of the sensor. Previous work by Roke et al.[1], has shown that the TACTIP is capable of tumour detection in the context of remote palpation of artificial flesh. What differs here is that the TACTIP sensor will be dragged along the target surface rather than palpated.

In previous work, [2] we have shown that the TACTIP is capable of texture analysis whilst being dragged along a textured surface. It is the application of tumour detection and lateral movement along a target surface that we propose to enrich the detection capabilities of WCE.

\section{Previous Work}

TACTIP is a biologically-inspired sensing device, based upon the deformation of the epidermal layers of the human skin. Deformation from device-object interaction is measured optically by tracking the movement of internal papillae pins on the inside of the device skin. These papillae pins are representative of the intermediate epidermal ridges of the skin, whose static and dynamic displacement are normally detected through the skin's mechanoreceptors, see Fig.1.

PillCam $\AA$ capsule endoscopy is a commercial product used within the medical industry as a minimally invasive endoscopy tool. The pill is swallowed by the patient and an internal camera records the journey through the GI tract. Spada 


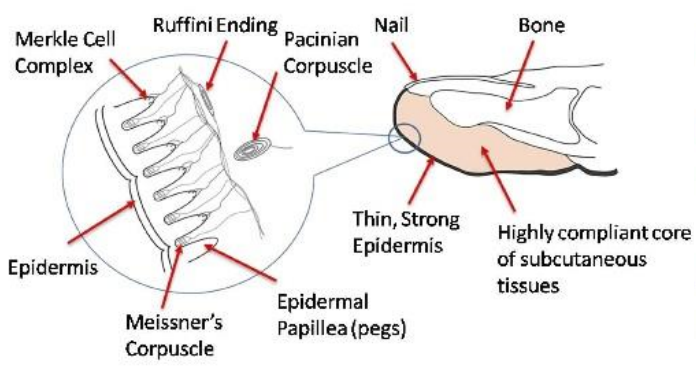

A.

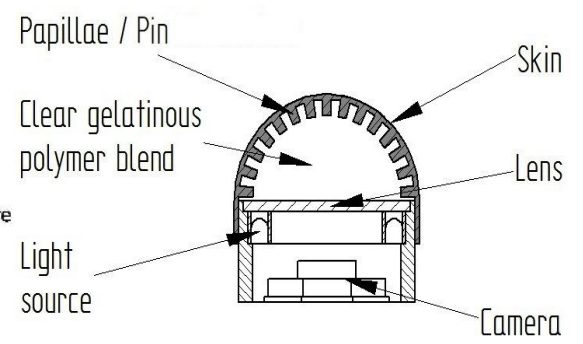

B.

Fig. 1: A. Biological inspiration for TACTIP, B. TACTIP tactile sensing device.

et al. report a case study [3] identifying the advantages of the PillCam over traditional endoscopy. The work concludes that the PillCam can be a feasible and safe diagnostic tool, and may represent an alternative in cases of difficult or incomplete colonoscopies, however Triantafyllou et al. [4] evaluate whether WCE can complete colon examination after failure of conventional colonoscopy. Their findings conclude that in their series of patients with incomplete colonoscopy, WCE did not always satisfactorily examine the colon due to insufficient exposure to the entire region at risk.

\section{Methods and results}

Fig.2 demonstrates the concept of placing a TACTIP sensor around the surface of a capsule endoscope. As the capsule passes naturally through the GI tract, the walls of the capsule push against the intestinal wall which will stimulate the TACTIP sensor. As far as we know little or no work has been published exploring such a tactile sensor on a capsule endoscope with the intention of tumour detection.

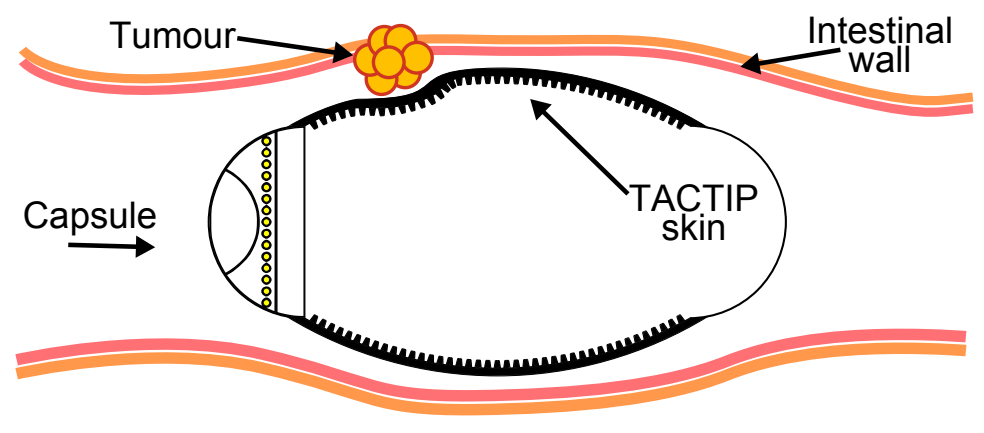

Fig. 2: Tactile sensing capsule endoscope.

An initial proof of concept experiment has been performed whereby a TACTIP sensor has been laterally dragged along the surface of an artificial tissue sample. Embedded within the tissue sample is an artificial tumour. The density of the tumour is much greater than the tissue and so it leaves an impression on 
the TACTIP surface which is easily identifiable with image processing as presented by Assaf et al. [5]. Fig.3 demonstrates the internal TACTIP view with the output from the image processing algorithm overlaid over the raw camera image. The three images show progression of the TACTIP across the tissue sample with the identified tumour moving right to left.

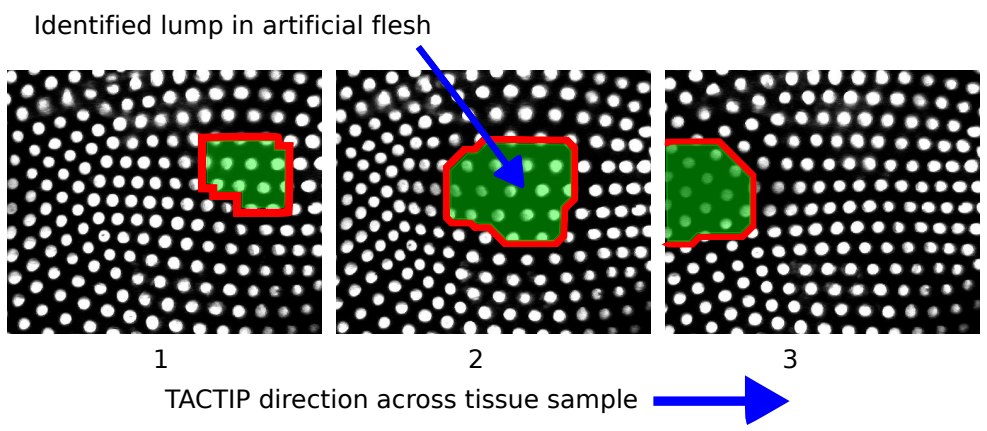

Fig. 3: TACTIP demonstrating lump detection. The TACTIP is laterally pushed along an artificial tissue sample from left to right and the embedded tumour is shown moving right to left.

\section{Summary}

Our initial experiment has shown that the TACTIP sensor has potential as a tactile sensor for capsule endoscopy with the ability to detect tumour like objects embedded in soft tissue wall. With some changes to the TACTIP design the device could be reshaped to fit on to the exterior of a capsule. This would offer either an alternative to the standard image camera, or an additional sensing technology to enrich overall data acquisition.

\section{References}

[1] Roke, C., Spiers, a., Pipe, T., Melhuish, C.: The effects of laterotactile information on lump localization through a teletaction system. 2013 World Haptics Conference (WHC) (April 2013) 365-370

[2] Winstone, B., Griffiths, G., Pipe, T., Rossiter, J.: TACTIP - Tactile Fingertip Device, Texture Analysis Through Optical Tracking of Skin Features. In: Living Machines. (2013)

[3] Spada, C., Riccioni, M.E., Petruzziello, L., Marchese, M., Urgesi, R., Costamagna, G.: The new PillCam Colon capsule: difficult colonoscopy? No longer a problem? Gastrointestinal endoscopy 68(4) (October 2008) 807-8

[4] Triantafyllou, K., Tsibouris, P., Kalantzis, C., Papaxoinis, K., Kalli, T., Kalantzis, N., Ladas, S.D.: PillCam Colon capsule endoscopy does not always complement incomplete colonoscopy. Gastrointestinal endoscopy 69(3 Pt 1) (March 2009) 572-6

[5] Assaf, T., Chorley, C., Rossiter, J., Pipe, T., Stefanini, C., Melhuish, C.: Realtime Processing of a Biologically Inspired Tactile Sensor for Edge Following and Shape Recognition. TAROS (2010) 